\title{
A Mathematical Theory of Language
}

\author{
Hans Gua \\ Correspondence: Hans Gua, Yeda Village, Xiyu Town, Xihe County, Longnan, Gansu 742100, China.
}

Received: November 8, 2017

doi:10.11114/ijce.v1i1.2893

\author{
Accepted: December 24, 2017 Online Published: December 27, 2017 \\ URL: https://doi.org/10.11114/ijce.v1i1.2893
}

\begin{abstract}
Modern linguistics cannot define and identify the best or standard pronunciations, writing and grammar. The choice and decision of sole human unified standard official or common language cannot be solved by modern linguistics generally. The current linguistics is no longer met the human developments because it can't answer what the best language is. The sole unified standard official global English cannot appear because of English linguistic level and shortcoming mainly. English linguistics comes to predominate in the contemporary era. Negating and improving the current linguistics must be negated and improved English linguistics first. The finite numerals are expressed infinite quantities in the mathematics. The finite sounds are represented the infinite meanings in the language. The theory and method are almost same in the mathematics and linguistics generally. The linguistics is a branch or concrete application of information theory (IT). IT is based on the probability theory and statistics generally. A meaning is often certain code, string of codes or mathematical value in the language. Defining or explaining certain meaning of language is measured and calculated a mathematical value actually. A subsystem or subtopic such as language teaching is often based on the general linguistics.
\end{abstract}

Keywords: mathematical theory, language, linguistics, human common language, mathematical value, meaning of language

\section{Introduction}

What are the best or standard pronunciations, writing and grammar? What is the best language? Modern linguistics cannot solve these problems. British English is decided by some educated people and Royal family in the southern England. Some American people considered that American English is differed from British English because of some new words. In facts, the sounds, writing and grammar of American English almost cannot change British English ones at all. The UK and US are squabbling over English language should be decided by native speakers themselves merely. Some real Englishmen are angry that some British English usages are illegal on the internet such as MS-word 2007 and OneNote. The requirement and claim of American English, British English, Australian English and other Englishes can lead the different national or geographical Englishes, that is, different English dialects, not sole unified official global English. It seems that the earth is smaller and smaller. All living languages are often crowded together now. The radio, TV and internet, especially global economy, trade, religion and etc are platforms which languages can behave, compare, learn, invade, improve and compete each other. The need to use sole human unified standard official or common language is becoming more and more pressing in the world. The defects of any living language are that its sounds, writing and grammar are too complex and far from the standard. One standard of English ASCII (American Standard Code for Information Interchange, ISO-646) is too many and far from the requirement of standard. Chinese GB2312-80, TCA-CNS 11643 (BIG-5) and Japanese ISO-2022-JP are much more complex and more than ASCII. The ideal human common language must be created new one. The human common language must will be used the standard sound, writing and grammar. The present linguistics is no longer met the human developments. The English linguistics is the dominant status in the present era generally. The current linguistics especially English linguistics must be negated and improved. A subsystem or subtopic such as language teaching, the choices of teaching materials and methods are often based on the general linguistics.

\section{Method}

A language is a complicated entity, likes any other thing. A sound, a word, a language and a meaning can be dealt in the same ways according to problem size, accurate degree, system theory, mathematics and etc. Any meaning or thing must be expressed by limited sounds in the language. The language and meaning are so closely linked that a lot of people often consider that their language or dialect is thought or meaning. Any language can use finite sounds to express 
infinite meanings normally. Any complex meaning can be expressed by the language. We often unite and separate certain meaning according to situation. A meaning is often a code, string of codes or mathematical value in the language. That is, the complex reality or meaning can be represented by the few or finite sounds in a language. Any reason or claim must be become some voices in any language. It happens frequently that combining and analyzing meaningful units or meanings are difficult, likes the multiple options or error operations of a computer. For examples, be, half and behalf, black, bird and blackbird, man, date and mandate, as, certain and ascertain, be, loved and beloved, in, voice and invoice, in, so, far and insofar, what, sit, what, s, it and whatsit in the oral English. Any language is often a complex meaningful system, liked a computer. How to learn and use a language is often a technique and skill for individuals. Learning and using a language is liked as learning and using a computer. Measurement and evaluation of a language is almost same as the measurement and evaluation of a computer. Certain nation and its language are used a same form or word in the English generally. In fact, a language has almost nothing to do with its users. English language is no longer tied its people and used by Englishmen only. It is a lie that French culture can be represented by its language only. French culture such as the fashion of Paris can be spoken or represented by almost any living language. The UNESCO (United Nations Educational, Scientific, and Cultural Organization) are helping the French to complete with other languages especially English in the world. In facts, some Frenchmen are wanting their language to defeat the other languages and popularise all over the world. The requirements and conditions of language for illiterates, college students, scholars and many other people are often different. The need to learn and understand meanings is becoming more important than the languages in the different speech communities generally. English uses the sound sequence [dog] to refer to an animal of a particular species while Chinese uses [gou] to denote the same animal. But mom is almost same speech sounds and meanings in the Chinese and English though they are different languages completely. Of course, the same pronunciation of mom is considered 1 syllable by Englishmen, 2 syllables by Chinese people. The language is very important for human, but we cannot aggrandize its function and characteristics. The language can serve the human as large as possible according to its features or characterizations. The feature or fatal shortcoming of the language is that any language is some human sounds merely. The deaf mutes cannot use any natural language and have to communicate with other tools generally. Any meaning or language is the description or presentation of reality, but not reality which be intend to represent by itself. For instances, it is funny to sentence one's death according to what one said he had killed a person. It is often unreasonable and even stupid that people should and must speak French because of French culture. Simply, the language is a man-made tool or system. Any language can express the abstract and inexistent things. The main tasks of linguists are researches how to express almost any meaning in the language, but can't decide whether or not a meaning is true. Whether a meaning of language is precise or true is decided by certain condition or user generally.

The human common language is selected in all living languages first. A national or regional official language can become human common language at the biggest possibility. It is strongly dependent upon the 12 world's top standard official languages which are 6 working languages of the UN and 10 most spoken languages with population estimates from the Ethnologue (18th edition 2015). The 12 world's top official languages are Chinese, Spanish, English, Arabic, Hindi, Bengali, Portuguese, Russian, Japanese, Lahnda, German, and French. The living languages especially the official languages are often eliminated or defeated many other languages or dialects in a country or region. The 12 world's top languages are often considered the best and most competitive languages in the world, and can reflect the level of whole human civilizations in general. English is the best among 12 world's top languages. English is an official language in the largest number of countries, the top language of second language (L2) speakers and internet (Wikipedia 2017). English writing is the best among 12 world's top languages. English typewriter is the best and cheapest in the world. English letters are monopolized the keyboard of computer. Any living writing must be translated into decimal numerals or English letters in the computer first. English is the smallest cost and shortest for antiilliteracy. Simply, English is the best and most competitive language among all living languages. It seems that English is the best candidate of human common language. If English was accepted as sole human common language, there isn't an unified official global English or sole standard English now. Of course, English must be localized, just as English language was in the Australia and USA if English is wanted to popularise in the China and India. But the localization cannot be affected and even prevented standardization. A lot of Indians often consider English is a foreign language though English has spread widely in the India. It is often misunderstood that American English and British English are belonged different languages and American English is the standard of English only. American English is first is weakening the competitive force of English, because American English is often excluded British English, Australian English and etc. The English journals are often accepted American and British styles and seemed to exclude Australian and other Englishes. Tom McArthur was proud of 'China syndrome' which English is popular in China, and seemed to unknow or accept that the US always negated British English and social managements (English Today. April 2006). English Today accepts American and British English at the same time and seems to negate other Englishes. The UK doesn't adopt or doesn't want to adopt American English as its standard. The French, Chinese and numerous other 
governments were intended to popularize their languages respectively all over the world. Their results are often obvious.

Measurement and evaluation of language system performance and efficiency are difficult due to the complexity of the internal structure of language system and because of the difficulty of describing and predicting the task. A language system is composed of subsystems, each of which can be viewed as a system with its own task and performance. Total system performance and efficiency are related to the performance and efficiency of the subsystems, although the relationship can be complex. Both language system and subsystem performance and efficiency measure the cost of learning and using a language generally.

The cost of learning and using a language is large generally. Mastering the pairings of sound and meaning or a language often needs a long term. Learners of the first or native language (L1) spend years learning a language, and still wind up with bad grammar and a funny accent. They must go to school. A lot of speakers cannot learn their native languages or dialects when they go to school today. There are not writings or textbooks in most of the world's living languages. Numerous people have to learn certain official language after they enter the school in the modern era. The official languages such as the standard Chinese, standard English and standard Russian are the smallest cost and difficult for communications in a country or region, neglected or negated any other requirement or claim such as politics, race, culture, religion, geography, age, gender, profession, social status and etc in general. The usages of 2 languages are more cost and difficulty than 1 (common) language between 2 speech communities. Recovering and developing economy rapidly is related to the Chinese policy of popularising sole standard language strongly though there are many different languages in the mainland China. Learning and using L2 are often worse than L1. The process of mastering L2, L3, L4 and even more languages respectively are almost same as L1. Using a language repeatedly is the reinforcing process of connection between meaning and sound. The more chances a language uses, the better the language you know is generally. Learning any language is often nasty, brutish and long. It is a lie that native speakers of English and Chinese can both learn Esperanto to fluency in 4 months. The fluent Esperanto did not mean to master its meaning and use accurately and flexibly in 4 months. Learned any official language such as English 5 years, learners of L2 were native primary school graduates at largest generally. After the gate of China was opened by itself, the other languages especial English is sweeping the mainland China. Several Chinese have become the rich men because of teaching English. The Craze English is misled that some Chinese people can master English in a short term. English has already been the most prestigious language in most universities of China. Most educated Chinese prefer English generally. Popularising English is even faster than Hong Kong and Taiwan in mainland China. It is often a fashion that young Chinese parents send their children to learn English and standard Chinese in the kindergarten. Many Chinese people are afraid that English will be replaced of its own language. Numerous French people just as other nationalities often prefer their native dialect or language and no choice to select language sometimes. Most Chinese often appreciate the fashion of Paris but don't appreciate French language. I have learned English over 30 years. Some basic skills of my English are much worse than a native English primary school graduate until today. The meaning almost has nothing to do with the language. But any meaning can and must be tied a media such as the language. Here, I must express my argument or meaning accurately and simply as a native English speaker as possible. Learning and using 3 or 4 languages are more difficult and even impossible for a person at same time. Few people can master over 2 languages. The efficiency of Singaporean or Swiss government is lower because of their 4 official languages respectively. The cost and difficulty of European Union are bigger because of its policy of language. It is better that English will be become a sole official language in the Canada. 3 official languages of the ISO and 6 working languages of the UN are too many, because the same meaning must be presented by 3 or 6 languages at same time. The Chinese and American policies of popularising sole official language strongly are better than Indian and Canadian ones. The global issues need a global language, especially a human unified standard official or common language. Languages which are difficult or expensive to use the internet will be declined first. Most of the world's languages which don't have writings or textbooks will disappear first. It is the smallest cost and difficult to communicate if the whole humanity could use the sole human common language. The standard sounds, writing and grammar must be dealt with the basic mathematical or philosophic problems first, for examples, simple and complex, absolute and relative, finite and infinite, discrete and continuous, unclear and accurate, and etc. 4 skills of mastering a language, speaking and listening are related speech sounds mainly; reading and writing are decided by the writing generally.

Modern language systems offer a number of options in terms of sound, writing and grammar configuration which allow wide flexibility in tailoring a given prescription to the task and the desired performance characteristics. Some examples of the options are speaking, listening, writing, reading, typing, typesetting, printing, meaning inquiring such as dictionary ordering and consultation, and etc. Sounds and writing are the hardware of language; grammar is the software of language. System turning refers to the optimization of grammar-related options, and reconfiguration refers to the sound and writing aspects. 
The Swiss Ferdinand de Saussure had proved that language was only a system of pure values with his methods or theories (Course in General Linguistics. Eds. C. Bally and A. Sechehaye. 1916). The linguistic method of postulates (that is, assumptions or axioms) and definitions is fully adequate to mathematics in the paper 'A Set of Postulates for the Science of Language' (Leonard Bloomfield. Language Vol. 2. 1926). The Danish linguist Louis Hjelmslev had presented more complete and comprehensive mathematical theory of language, such as simplicity principle, principle of analysis, first-degree derivate, segment, part and member, class, chain and paradigm, and etc (Prolegomena to a theory of Language. 1943). It is not always easy to see how much of this abstract theorising could be put into practice and it could be argued that, had Hjelmslev used fewer and a less individualistic terms, his theory might have been better and more widely understood. That is, few linguists understood and noticed the mathematical theory of language of Hjelmslev. The American Whorf thinked that languages dissected nature differently. The formulations of mathematics and of formal logic have acquired the reputation of dealing with this order of things: i.e. with the realm and laws of pure thought (Linguistic Relative. Science and Linguistics in Language. Thought and Reality: Selected Writings of Benjamin Lee Whorf. J. B. Carroll ed. MIT Press. 1956). Numerous aspects of Hjelmslev's methods are much more complete than the Chomskyan mathematical theory of language such as deep and surface structures. The American Avram Noam Chomsky couldn't breakthrough limitation in the Saussure's mathematical theory of language. The Chomskyan linguistic methods were reached mathematical theoretical level of language of Saussure at largest. The fatal shortcoming is that Chomskyan standard theory is based on the English only, for instance, whether or not a grammatical English sentence must be accepted or decided by a native English speaker. English should be become 2 different languages in the UK and USA respectively according to Chomsky's arguments and notions. The standard theory of transformational generative grammar was the revised edition of parole and langue of Saussure actually. In facts, any living language can express almost any meaning without English morphology and syntax. Chomsky had already recognised which and where the problems of his linguistic theories were, but he didn't know how to revise and unify his numerous linguistic notions and theories because Chomsky cannot understand the Hjelmslev's mathematical theory of language thoroughly until now. The Chomskyan revolution and standard theory are a lie completely. Chomsky didn't know how to modify his linguistic methods in the end, for instances, standard theory, extended standard theory, revised extended standard theory and etc. Chomskyan standard theory was impossible to become a linguistic 'bible' and universal grammar within English frame merely. I'm sure that Chomsky is impossible to understand my linguistic theory. The linguistic theory is more accurate and complete than Hjelmslev's mathematical theory of language at least and based on mathematics, philosophy and almost any related science in this paper. Simply, Chomsky didn't understand and master that his linguistic theories were solved by mathematical methods completely and comprehensively, though mathematics and philosophy were mentioned many times in his numerous linguistic works such as 'Syntactic Structures' (1957), 'Rules and Representations' (1980). Harris compared English and Hidasta, a Siouan language of north Dakota, concluded the limitations of procedures for linguistic description. The formulae can be used as a source of information on the grammatical meaning of the morpheme classes symbolized in them. The utterance formulae are thus rather like the formulae for the phonetic structure of a language, and even like phonemic writing: all of these are formulae showing what occurs in the language. If we now seek a clearer method for obtaining generalizations about the structure of utterances in a language, it should preferably deal with the simplest observables (From Morpheme to Utterance. Zellig Sabbettai Harris. Language Vol. 22. 1946). That is to say, Chomsky has almost backed the mathematical theory of language of Harris.

Any speech sound is a complex continuous entity, liked the binary signal or code of computer. A language is a computer. A language is measured and calculated the meaning or reality generally. The finite sounds express the infinite combinations or information in a language. The sounds are the hardware of language. The meaningful units such as words and discourses are the software for language generally. The human language can be improved according to the computer science. For example, any meaningful unit of the language is a code or string of codes. Not only the language can be expressed or calculated by a computer, but also the other things such as picture and philosophy. Simply, the linguistics is almost mathematics. Vice versa, the standard of speech sounds can calculate and prove $1+1=2$ actually. For instance, what is 1 sound? That is, what is 1? Any continuous sound must be discretized and linearized in any language generally. A standardized or prescribed sound must be become an actual pronunciation which has a large number of variations or approximate sounds in the language. The English and Chinese [a] has numerous different [a]s in the actual communications, such as different length, stress, pitch and etc. The scope and property of certain sound such as [a] is very large in the English and IPA. We don't know how to pronounce and identify them sometimes. But any language must be defined and identified its every sound at first. The language expresses meanings is how the mathematics represents the numeral values, accounts abstract quantities, like time, temperature, electricity and opinion. More simply, linguistics is the same theory how a computer works. The human sounds are symbolized, that is, numbered. Decimal integers are corresponded to the syllables of language normally. The random variables are syllables in the meaningful units of language generally. Mathematics calculates the numeral values. Linguistics studies or 
calculates the meaning. Certain meaning is certain numeral value. Any meaning of language is the approximate value of real world. You measure or calculate the approximate value of the real world according to condition actually when you want to express certain meaning exactly. Of course, maybe you don't know or concern. Whether the meaning of certain unit of a language is accurate and correct is decided by the requirement and condition generally. The translation of language B is the approximate value or meaning of language A. Although my English is much worse than Chinese and I often confronted unknown English words, recognized and understood the arguments or terms of the original A COURSE IN MODERN LINGUISTICS (Macmillan. 1958) which is written by C. F. Hockett is much better than its Chinese translation (She Zhenyu \& Ye Feisheng. 1986. Peking University Press. No. 9209.53).Certain grammar of language is almost certain algorithm of computer. For instance, English morphology and syntax are certain addition, subtraction, multiplication and division of values or meanings. Mathematical logic can be referred. The human recognizes language is just liked that the blind men touch an elephant. In fact, a man who can see often doesn't know or tell what an elephant is. It is often difficult to describe and prescribe an elephant or sound. An English syllable can be read 2, 3 and even more sounds by Chinese people. A Chinese syllable can be considered and pronounced 2 sounds by some Englishmen. Simply, an English syllable can have 2, 3 or 4 Chinese syllables. 1 Chinese syllable may have 2 or 3 English ones. My English name wasn't allowed to use by Bank of China. My name has 3 Chinese sounds or syllables. I don't know how my Chinese name GUO XIAOKUAN is spoken by Englishmen. English phonetics which likes a blind man touches the ear of elephant. Chinese phonology touches its leg. Japanese phonetics is touching upon its tail. A sound or elephant is almost not known or defined in modern linguistics. This is why the International Phonetic Alphabet (IPA) has some theoretic values but few practical values. It is often false and null hypothesis that the general principle of current IPA is to provide one letter for each distinctive sound. For instance, whether or not 1 long vowel is 2 short vowels and a syllable is a sound. What is a speech sound is one of the core issues in linguistics, especially phonetics. The meaningful units of a language must have a syllable or sound at least. The silence is often excluded from language. Linguistics is depended on the other sciences or human level of civilizations. Any sound of language is a man-made or theoretic value or symbol. The actual pronunciations vary somewhat from phonetic notations respectively in any language. The distinction of English phonetics and phonology loses the base of existence after the IPA appeared. 2 English words of phonetics and phonology are 1 Chinese word or meaning. Some Englishmen are often proud of knowing the exact differences between phonetics and phonology. In fact, one English term of phonetics and phonology is an unusable word according to Chinese. Certain problem must be appeared that English phonetics cannot suit Chinese or Japanese sounds, or Chinese and Japanese phonetics cannot explain and define some English sounds. Such problem cannot be solved by the IPA and current linguistics. Any phonetics can be tested in the actual oral communication. The empirical test is one way of the measurement and evaluation of speech sounds. The more samples tests are, the more reliable results are according to the statistics. Any speech sound is included infinite features or variants. For examples, long and short, strong and weak, round and unround, qualities of sounds, positions and shapes of speech organs, and etc. Sounds of any language are often unstable in the actual communications. You often can perceive the difference of 2 same syllables between 2 persons or 2 times of a person in any language. People have to focus or express 1 or some features of 1 sound at a time. There are intonations in almost every language. Any definition or explanation of certain phonetic term is liked as the approximate value in the Mathematics. The definition or explanation can approach infinitely to its term. Whether or not a definition is accurate is decided by the condition or precision generally. The language is a man-made tool. The problem is always being solved by human that the language especially the sound and writing is as easy to learn and use as possible, as small cost as possible, as large scope as possible, and etc. Theory and practice can develop with each other. It is customary to assume that a phoneme is the smallest unit and syllables are the biggest units in the sounds. In fact, any smallest unit, that is, any phoneme can be divided forever. The biggest units such as syllables may group forever. For examples, an English vowel has 2 variables, long and short in general. A Lahnda consonant has 3 variables, 3 tones. A Chinese vowel has 4 variables, 4 tones. A Vietnamese vowel has 6 variables, 6 tones. British English has 24 consonants and 20 vowels. The current IPA has 39 vowels. In fact, vowels can be classified into 2, 3, 4, more and even infinite according to the current IPA chart. Any speech sound often has a theoretical ideal point or value and $\mathrm{m}$ related actual pronunciations or approximate phonetic values. The speech sounds are social statistical results usually. That is, the speech sounds are public productions which cannot be decided by one or some people. Any sound is allowed the very extensive scope in order to fit that the language is used as more people as possible. Any writing and sound requirement or claim of individuals must be obeyed the social behave or requirement unconditional. That is, the standard sounds and writing must be ignored some requirements and claims of individual person or language. For example, the standard British English or its sounds must be negated or ignored some requirements or claims of the educated people and royal family in the southern England. Simply, any human claim, requirement or meaning must be become some voices or sounds in the language. Any meaning of language is certain speech sounds. Certain speech sounds must be evaluated and measured speech activities or behaviors, and etc. Evaluating and measuring meaning of any language are evaluated and measured speech sounds first. 
The sounds and writing of a language can be improved according to the information theory (IT), especially, the coding theory. IT, however, does not consider message importance or meaning. 2 completely same meaning and thing are almost not existed. But the language does expresses meaning and language A often can be translated language B. English spoken and written messages are different meaningful systems according to IT. Of course, the writing can overcome some shortcomings of speech sounds. Any semantic feature or rule such as the grammar must be obeyed the phonetic principles first. That is, any requirement or claim of language especially for its sounds cannot be conflicted with the axioms of IT at least. The linguistics is a branch or concrete application of IT. The language is one tool of many communications. A language is almost same as the traffic light, mechanical and electronic codes according to IT. The language always changes and develops. The living languages especial natural languages are not natural at all. Languages are always invaded and competed each other when they meet together. Chinese and American governments have sought to promulgate the standards of language repeatedly and are promoting to popularise their own languages respectively all over the world. The speech sounds almost have nothing to do with any meaningful system or grammar. If A. N. Chomsky considered the Chinese or Japanese grammar, perhaps the transformational generative grammar could disappear. The Chinese and Japanese have negated the numerous aspects of Chomskyan standard theory, such as morphology, case grammar and etc. Case and gender of German, French, Spanish and Russian are more complex and more than English generally. 'The grammar of English nominations' (Chomsky, 1960) is produced a large number of unusable rubbish words according to Chinese. For examples, English 'action' is a redundant word according to Chinese, which can be represented by 'act' directly in the Chinese. But the few English verbs have the same meaningful nouns respectively, e.g., approach, substitute and model. The standard theory is that any grammar cannot be contradicted with the phonetic rules unconditionally. The English 'the' is a meaningless and superfluous rubbish word. Perhaps the using rate of 'the' is the highest in the English. There are a lot of the in my paper. Can English grammar solve such problem? Or can 'the' be removed from English? The English articles such as a and an are redundant rubbish according to Chinese. Simply, English sounds and grammars are no longer the standard of language. English 'linguistics' is a superfluous and unusable word according to Chinese. English linguistics is represented by the science of language in the Chinese. Of course, linguist is expressed the scholar of language. Mathematician is called the mathematical scholar by Chinese.

One defect is that English sounds are unequal long in its standard sounds. It is stranger and stupid vague theory that some English vowels such as semi-vowels $[\mathrm{y}]$ and $[\mathrm{w}]$ are belonged consonants. Each of $[\mathrm{y}]$ and $[\mathrm{w}]$ does form a syllable respectively; some English consonants such as [ky] and [ty] are Chinese syllables; [ $\mathrm{n}]$ is a Chinese and Japanese syllable, but English prescribes its consonants cannot become syllables. According to English criterion, a consonant is long as a semi-vowel or half length of a vowel, i.e., a phoneme [u] is longer than [w]. A phoneme or syllable [u:] is longer than $[\mathrm{u}]$. [a] is longer than $[\Lambda]$. [au] is longer than [ua] [a] or [u]; [a] or [u] within [au] is shorter than a single vowel if a diphthong [au] is pronounced same long as a single vowel. [if] is longer than [i] or [f]. [aiə] is longer than [ai] [iə] [a] [i] or [ə]. [st] is longer than [s] or [t]; [s] and [t] of [st] are shorter than a single consonant respectively if a consonant cluster [st] is long as a single consonant. [kst] is longer than [ks] [st] [k] [s] or [t]. [bed] is longer than [be] [ed] [b] [e] or [d]. [best] is longer than [bes] [st] [be] [es] [b] [e] [s] or [t]. Some phonemes and most syllables are false and null hypotheses or entities in the English. French, German and Spanish sounds are more than English according to their criteria respectively. The fatal shortcomings of IPA are that phonemes are too many and ambiguous, but no syllables. The dream of standardized sound representations of some English and French linguists can't become the reality until today because of English phonetics and linguistics mainly. English sounds are often decided by Englishmen randomly. For examples, the first element of an English diphthong is strong, clear and distinct; the second element is rather weak and unclear. The first consonant must be said very gently and quickly, and the second one is very often formed while the first one is being pronounced. English $[\Lambda]$ isn't the consonant, but $[\Lambda]$ is the semi-vowel of [a] according to its criterion. English pronunciations are often unstable, unreliable and uncertain in its phonetic system. The long and stress are often excluded from Chinese and Japanese sounds. Chinese and Japanese sounds are equal long codes generally.

Syllables are the random variables within a meaningful unit and phonemes are restricted within a syllable in some languages such as Chinese and Japanese. Syllables are not random variables in the meaningful units such as English, Spanish, French, German and Russian. English syllable theory is often complicated and confused. The phonemes are the random variables in the most English syllables, but the combinations or appearances of some phonemes and syllables are restricted. [au] is a diphthong or syllable. A phoneme or syllable [a] can't appear after [u]. A syllable [ua] cannot be existed in the English and Russian. The essential of English phonetics or an English fatal weakness is that sounds are often the conditional variables. Syllables are often equal long and random codes in the Chinese and Japanese. Numerous aspects of Vietnamese are worse to relate to English phonetics and linguistics. Modern Vietnamese is complicated Chinese tones and English letters. The number of Japanese sounds are too small, 5 vowels and no more than 113 syllables, but the misheard or error rate of Japanese sounds is the smallest among 12 world's top languages. Another shortcoming of English is that the syllables are often changed and reduced whenever English words are 
combined the larger meaningful units such as phrases and sentences according to its criterion.

Some English and French persons wanted to standardize speech sounds, but English and Russian phonetics and linguistics are being stopped to standardize sounds. You can't test English sounds if you are not able to speak or understand it according to its morphological rules. Numerous prescriptions of grammar especially morphology about sounds must be negated or deleted. I must negate the claim of British linguist J. R. Firth: "Without phonetics there can be no morphology of a spoken language, without intonation no syntax" (An Introduction to English Phonetics. 1984). The sounds of any language whether or not someone understood can be used and tested by individuals. For examples, $[\Lambda]$ is longer and stronger than [a] in the English sometimes, which can be tested by listening to native English speakers. [ $\Lambda$ ] and [a] must be eliminated one of them. [ $\Lambda$ ] and [a] will be coalesced into a same phoneme in the standard sounds or broad transcriptions of new IPA. The most Chinese, even educated people often confuse 4 tones of the same Chinese initial and final. Simply, the human standard sounds are no longer coded according to any current phonetics or linguistics.

English letters are random variables generally. Chinese characters and syllables are too many and complex. Compared between English and Chinese, some shortcomings of each are obvious. The syllable [i] must be spelled [yi], the syllable [u] must be [wu], and etc in the Chinese. Chinese character and Japanese kana don't need any other symbol to separate sounds, but are too many. Although English letters are simple and convenient, some sounds are awkward to separate and identify. Some syllables must be separated by an other symbol at least. Chinese, Arab, Hindi, Bengali and Lahnda writings are integrated with some shortcomings of English and Japanese, and much worse than Spanish, English, Portuguese, Russian, Japanese, German and French writings. However, Hindi and Bengali writings are much better than Chinese, Arab and Lahnda ones. This is one of challenges for the human common language. The human common writing must concentrate advantages of each such as letters, Chinese characters, Japanese kana and etc respectively, overcome their shortcomings. The problem is how sound and writing are the optimal matched each other according to modern human requirements. In fact, most writings have already united by the binary letters.

Most world's writings are no longer treasures but barriers to communicate between global citizens. Most writings are often the different codes of same human sounds and meaning. The symbols of Unicode such as ASCII (ISO-646) are too many and complex. We must improve or change our language so as to meet and promote the whole human developments. The English writing is much worse than the ideal, standard or future human common writing though English typewriter is the cheapest and best in the world nowadays. That is, the Latin and Greek alphabet are no longer satisfied the requirement of modern human writing, for instance, the shape and number of Latin and Greek letters are far from the requirement of standard. English writing is being become more complex, but not simpler. For examples, @, \&, $\$, \%$, the mark of case grammar ' and etc are being become letters in the English actually. French, German, Spanish, Portuguese and etc are being improved their writings according to English writing or criterion respectively. Chinese, Arabic, Hindi, Bengali, Japanese, Lahnda writing and etc are primary ones which can satisfy the brush and pen only, their mechanical and electronic products, dictionary retrieval and social managements are much more expensive and difficult than English letters. English letters are occupied the keyboard of computer because other writings such as Russian letters are more and worse than English ones. Although the human writings have already been unified by the computer, the binary letters of a word are too long to learn and use for the ordinary people.

The handwriting can be fitted almost any writing in existence. The cost and performance of English and Russian typewriters are much better than Chinese, Japanese, Hindi, Bengali, Arabic and Lahnda ones. Typewriters aren't popular until today because most of the world's writings are too complex. Easier Learned and used the writing are, more restrictions the condition or technology of writing is. The current Latin or Greek scientific terms will be replaced by the human common writing first. Simply, the human common language must be matched the standard sounds and writing, the easiest for antiilliteracy, used the cheapest and best typewriter, especially for the mechanical typewriter foremost.

It is relatively easy to prescribe grammars. In practice, however, one wants not only grammar, one wants good grammars. Thus, the objective is to use good grammars and prove that they are good. The 'goodness' of a grammar can be appraised by a variety of criteria. One of the most important is the time taken to execute it. There are several aspects of such a time criterion. One might be concerned with the execution time required by different grammars for solution of particular problem on a particular language. However, such an empirical measure is strongly dependent upon both the prescriptions and the language used to implement the grammar. Thus, a change in a grammar may not represent a significant change in the underlying grammar but may, nevertheless, effect the speed of execution. Furthermore, if 2 grammars are compared, first on one language and then another, the comparisons may lead to different conclusions. Thus, while comparison of actual morphology and syntax running on real languages is an important source of information or meaning, the results are inevitably affected by grammar skill and language characteristics. The best grammar of a language is almost the best algorithm of a computer. Of course, the current algorithm of computer is much more accurate and complete than the grammar of language generally. 2 important aspects of a concise language 
are as follow: First, the most common words should be shorter than less common words so that sentences will not be too long. Such a tradeoff in word length is analogous to data compression and is the essential aspect of source coding. Second, if part of a sentence is unheard or misheard due to noise, e.g., a passing car, the listener should still be able to glean the meaning of the underlying message. Such robustness is as essential for an electronic communication system as it is for a language; properly building such robustness into communications is done by channel coding. Source coding and channel coding of computer can differ, but source and channel coding of language are same generally.

A useful alternative to such empirical measurements is a mathematical analysis of the intrinsic difficulty of solving a problem grammatically. Judiciously used, such an analysis provides an important means of evaluation the cost of grammar execution. Any meaning of language exists in certain time and space generally. English and Russian time and space complexities or grammars are more complex than Chinese and Japanese ones generally. It is often more easy to appear ungrammatical mistakes when one learns and uses English and Russian.

In contrast to Chinese, one of the fatal shortcomings is that English morphology and syntax are too complex and Japanese has concrete entities or patterns of sentences. There are few and even no morphological words in Chinese and Japanese. But any related morphological meaning can be expressed by Chinese and Japanese. Russian morphology and syntax are more complex and more than English, French, German, Spanish and etc in general. Charles Francis Hockett accounted: Mandarin Chinese scores very low, with barely more than one morpheme per word on the average. English shows nearer to two morphemes per word; Spanish about two and one half; Latin about three; and Fox nearly four (A Course in Modern Linguistics. 1958). Although the Esperanto which is one of the international auxiliary languages simplified morphology, its morphology does not disappear completely. 16 Esperanto grammar rules are coarse and ambiguous. For example, Esperanto cannot be proved that its 28 phonemes or letters are the best. Esperanto has no future. I often worried about inflection and concord when I learned and used English and Russian. Compared to Chinese, morphological words such as large quantities of tense and participle of English verbs are almost unusable rubbish, deteriorated the performance of English. English be has morphological words is, are, was, were, been, being and etc. Any exact meaning of is, are, was, were, been and being can be expressed by be in the Chinese. Is, are, was, were, been, being and etc are unusable words according to Chinese grammar. Simply, you master be only. It is unnecessary and redundant to learn and use is, are, was, were, been, being and etc in the Chinese. Is can be analyzed the present single be. Were the past plural be in the Chinese, and etc. There are ungrammatical meaningful units such as ungrammatical English phrases and sentences. There are not ungrammatical values or meanings at all. For instance, an ungrammatical English sentence 'The man come' is a grammatical Chinese sentence. In facts, the language can express inexistent things. One fatal weakness of English syntax is concord, that is, its words must be corresponded or matched each other according to certain morphological feature in the groups of meaningful units. In fact, the English syntax has nothing to do with its morphology. Some morphological words such as the third person he, she, they and it can be used directly by Chinese, without changed Chinese syntax. There aren't distinctions between they and them, he and him, she and her, I and me, and etc. Any exact meaning of they and them, he and him, she and her, I and me can be expressed with the same word of each pair respectively in the Chinese. Each of English his, her, theirs and its a phrase one of he, she, they or it and the antonym of of in the Chinese. That is, a large number of English words such as his, her, their, our and its are redundant and unusable according to Chinese. The essence of English morphology and syntax are same, for instance, ungentlemanliness. It seems that English morphological words are more concise and accurate than corresponded Chinese words. But morphological words often provide the superfluous information in the English sentences which manifest concrete time, person, gender, number and etc. Learned and used English are much easier if English morphology and syntax were disappeared or improved. That is, English can use Chinese grammar directly. Vice versa, if Chinese words used English grammar to form phrases and sentences, Chinese must be added large number of morphological words. The cost of learned and used Russian is much bigger than English, French, German and Spanish because of morphology generally. There are 6 Russian case grammars at least. The essence of complex English morphology and syntax is irregular in general, for instance, the numerals such as first, second, third, fourth and ith are unusable rubbish according to Chinese. They are often represented by No. 1, 2, 3, 4 and etc respectively in the Chinese. English words and their morphemes are same units or forms in the phrases and sentences actually though its words and morphemes are prescribed or defined the different hierarchical meaningful units. At least half of frequently English words are redundant and can be removed from current English according to Chinese grammar or mathematical logic. That is, the algorithms or arranges of English meaningful units or meanings are too awkward and complex. The smallest meaning is almost not existed at all. But the language must have a smallest meaningful unit. English number is often contradicted with the mathematical axioms or prescriptions directly and produced many dump of words, for examples, plural, uncountable and mass nouns, adjectives, and etc. The competence or number of Japanese sentences is limited greatly because of its concrete entities or models. Chinese and English sentences are often abstract entities. That is to say, there are more restrictions in the Japanese sentences. English case grammar such as people's is almost a stupid method or form. The pronunciation of people's is same as its plural form, and the punctuation ' is become a letter 
actually in its writing. People's is expressed by a phrase people and antonym of of in the Chinese. Simply, English case grammar is become antonymous meaning of of in the Chinese. Russian, French, Spanish, Arabic and German are much worse than English because of grammar. I often worried about and even hated Russian grammar. A lot of Russian grammatical rules or prescriptions are unreasonable and stupid. Of course, learning and using Russian are more cost and difficult than English. The Russians often fear and even hate Chinese characters when they learn the Chinese. Although the Arabic $0,1,2,3,4,5,6,7,8$ and 9 are the best decimal numbering symbol system in the world, its numeral words are often more complex than English and Russian. English morphology and syntax are complex, confusion and always unexpected. There is a strange phenomenon. Chinese does not have a word which is corresponded exactly to English of normally. There is not a word which is the antonym of of in the English generally. At least 3 Chinese words are often corresponded to the antonym of English of. It seems that English meanings have addition, no minus directly. Chinese has minus, no addition directly. Here, I can create a new English word de which is the antonym of of. English morphology and syntax are produced a large number of superfluous words or minimal meaningful units according to Chinese or mathematical logic. Simplifying English morphology and syntax can be referred Boolean calculations. For examples, un-, -tion, -ly, -less and etc can be removed or separated from the corresponding English words respectively. Simply, English words can use Chinese grammars to combine directly. The Chomskyan standard theory did not exist at all. Many Eskimo words of snow just as Arabian camels and Chinese horses can enter English or human common language directly. Chomskyan samples 'the man comes' and 'the men come' can be simplified 'the man/men come'. The performance and efficiency of a language are almost same as the measures of a computer. The numerous aspects of Chinese grammar are much better than English generally because the combinations of Chinese meanings are more regular or uniform, for examples, the expressions of comparatives and superlatives, past, present and future, and etc. Contrasted English, a large number of Chinese classifiers or measure words are unusable rubbish. For instance, Chinese 'a tree' must be added a unit or scale or measure word generally. Langue and parole of Ferdinand de Saussure, competence and performance, deep and surface structures of Chomsky are superfluous and unusable distinctions or terms according to mathematics and IT. The individuals can express the different or opposite meaning with same sounds and words in any language. Meaningful units such as words and sentences are independent but influenced each other in a language generally. The language was often conceived of as a structure of levels and interrelated parts and continuum. Dani speakers use only 2 words, mili(dark) and mola(light), to name all the colors of the spectrum. Proponents of the Sapir-Whorf hypothesis believe that Dani and English speakers perceive colors in different ways, but same according to IT. The human common language must be reduced and even avoided complex grammars, that is, used the best algorithm or grammar. Although the meanings can be expressed unclear or undefined, there are few and even no grammar errors in the human common language. A. N. Chomsky is a great linguist though his some views are often debated and criticized. He enjoyed the universal grammar and had already dealt some English meaningful units with the mathematical methods. The universal grammar of Chomsky was intended to prove that English was the standard of language. A meaningful unit or meaning such as a word or a sentence is certain mathematical value. The mathematical calculations are much more accurate and complete than current grammar, such as morphology, case grammar and X-bar theory. The standard grammar can be referred the computer algorithm especially mathematical calculations.

One of the considerations in the selection of a new language is the comparative cost/performance of the languages being considered. Judgments as to expected performance can be made informally based on the experience of others or the native speaker's claims. French government is intending to popularise its language in the world; but few people learn French in the China and India. In facts, English invasion cannot be prevented in the Russian and France. The computer English beyboard is the best advertisement in the world. TV5MONDE (Asia) is insisting to broadcast French, and shows English translation sometimes. German TV station DW stopped German channel, and shows English channel only on the No. 2 Asia-Pacific satellite. RT (Russian Today) is an English station. Muslim TV (Muslim Television Ahmadiyya International) often propagates Arabic religions in English. More formal studies involve experimentation using benchmarks or language models. Prediction of the task is an obvious problem if the language is being acquired for a new application or task. However, ever upgrading of existing language usually involves new functionality and features which have to be accounted for in the projected task. For instance, the population is 7.4 billion in the world now. A person must have a name at least. Certain naming custom such as Chinese or English one is no longer the norm. We must build a new naming tradition or standard. That is, the human names will be used according to the international standard in the human common language. The unified animal names are often decided by the animal association. No more than 1,000 words can be mastered in whole life of an illiteracy. Enormous programming languages just as English and Chinese are dealt different size of problems generally. Any global citizen who interests in the computer technologies must learn and master Mathematics and English generally. Optimization is a part of the selection problem since it is only reasonable to compare the cost/performance of languages which are turned to the task. 


\section{Results}

The mathematical or linguistic models used for this purpose require values for task parameters. Measurements from currently operational systems can give some insight into the range of tasks that might be expected for the new language system. Specific details of the task or meaning can be very difficult to predict since (1) the language may be used in many different environments and (2) the characteristics of the language can affect how it is used. However, general characteristics of sound, writing and grammar behavior have been isolated and found to be useful in language analysis, evaluation, test, use and design.

The Arabic numerals were defeated all other decimal writing system in the mathematics. The linguistics is almost same as mathematics. It isn't important perhaps who invented them though some people think that the decimal numerals of ASCII were invented by the Indians. The standard sounds, writing and grammar are restarted to define and classify according to modern human requirements and civilizations respectively.

Some fatal defects of English are clear according to modern human requirements of language. (1). Some English phonemes and most syllables are false entities. Some phonemes may silence in the actual pronunciations, as the b, t, k, l, $\mathrm{n}, \mathrm{w}, \mathrm{h}$, gh, etc. in lamb, listen, know, talk, autumn, answer, honour, fight, etc. (2). English alphabet or writing is far from the requirement of standard. (3). 26 letters cannot match English sounds one-to-one respectively. (4). English is decided by its native speakers merely, for example, English sounds are often decided by English people and even small parts of English people randomly. English fatal shortcomings are more obvious and sharp after the standard sounds, writing and grammar were established.

The French, German, Spanish, Portuguese and Russian are much worse than English because of letters, sounds, morphology and syntax. The French should be removed from 6 working languages of UN at first according to its elements, structures and speaker totals. French, German, Portuguese, Spanish, English and etc are dialects of certain language which has same writing according to the Chinese criterion; but some western scholars are insisting that some Chinese dialects are different languages in the China. For instance, the 10 top world's languages are Chinese, English, Spanish, Hindi, Cantonese, Bengali, Russian, Japanese, French and German according to total number of speakers in the UNESCO world report on languages (Sun Hongkai. 2001. On the Endangered Languages in China. Language Teaching and Linguistic Studies. Beijing Language and Culture University Press. ISSN 0257-9448). In facts, any living language should become the working languages of UN if satisfied the requirement of French.

\section{Discussion}

Any living language cannot be satisfied the modern human requirements of mechanization, electronization and digitization because of present linguistics. The ideal human common language is coded or created according to IT, mathematics, semiotics and relevant sciences. The living languages especially 12 top official languages are the experimental or raw materials of the standard sounds, writing, grammar and human common language. The human common language will be represented the fact or reality according to modern human requirements and civilizations.

Uniting and using the sole human common language is a long term strategy for whole human developments generally. Creating and popularising a new ideal human common language is often needed the long course in all human communities. English is the smallest cost and the largest performance among all living languages in the world generally. It's better to popularise English as sole human common language in the world now. Especially, English should be used as unique official language in Singapore, South Korea, India, Canada, Hong Kong, UN, EU, ISO, WTO and etc first.

\section{References}

Eugene, A. N. (1993). Language, Culture, and Translating. First edition. Shanghai Foreign Language Education Press. Shanghai. ISBN7-81009-722-9/H·364.

Gui, C. K. (1985). Applied Phonology of American English. Shanghai Foreign Language Education Press. Shanghai. No: 7218.072

Hans, G. (2017). The Standard of Speech Sounds. In, International Journal of Linguistics Vol. 9, No. 3. Macrothink Institute. Nevada US. https://doi.org/10.5296/ijl.v9i3.11446

He, S. F. (1985). Practical English Phonetics. Beijing Normal University Press. Beijing. ISBN7-303-00142-5/H·2.

He, Z. X., \& Mei, D. M. (1999). Modern Linguistics. Foreign Language Teaching and Research Press. Beijing. ISBN7-5600-1523-9/G.654.

Huang, B. R., \& Liao, X. D. (1991). Modern Mandarin. Higher Education Press. Beijing. ISBN7-04-010638-8

Information on the Internet, such as wikipedia.org, omniglot.com and etc.

Jiang, D., \& Qian, Y. M. (1992). Information Theory and Coding. First edition. University of Science and Technology 
of China Press. Hefei Anhui. ISBN7-312-00402-4/TN·15

Jiang, Y. J. (2003).English as a Chinese language. In Tom McArthur (Ed.), English Today, 74, 19(2). Cambridge University Press.

Liu, R. R., Stephen, R. M., Zhao, T., \& Yan, X. T. (1988). Readings in Linguistics: Seventy-five Years since Saussure. Mapping Press. Beijing. ISBN7-5030-0193-3/H·4.

Liu, Z. Y., Pen, X. D., \& Liu, W. L. (1993). Computer English. Tsinghua University Press. Beijing. ISBN7-302-01089-7/TP.408

Lu, G. Q. (1983). Modern English Lexicology. Shanghai Foreign Language Education Press. Shanghai. ISBN7-81009-029-1/H·019.

M. L. M. (2016). (Un)separated by a common language? Are American/British differences unimportant? In Clive Upton (Ed.), English Today 125, 32(1). Cambridge University Press.

Mei, L. C. (2017). An explicit pragmatic approach to integrative data analysis strategies for mixed methods research. In, International Journal of Linguistics, 9(3). Macrothink Institute. Nevada US. https://doi.org/10.5296/ijl.v9i3.11246

Raimo, A. (1989). Historical and Comparative Linguistics. University of California, Los Angeles. John Benjamins Publishing Company. Amsterdam/Philadelphia. ISBN 90-272-3557-0(pb).

Rodney, H. (1988). English grammar: An outline. Cambridge University Press. ISBN0-521-31152-7.

S. H. Burton. First published (1984). Reprinted in Hong Kong (1985). Mastering English Grammar. Macmillan Publishers Ltd. ISBN0-333-36368-X.

Wang, D. X. (1992). An Introduction to Sociolinguistics. First edition. Beijing Language and Culture University Press. ISBN7-5619-0159-3/G.45.

Ye, B. K. (1992). General Linguistics: A Survey. Xiamen University Press. Xiamen. ISBN7-5615-0443-8/H·32

Yin, B. Y., \& John, S. R. (1997). First edition. Modern Chinese Characters. Sinolingua. Beijing. ISBN7-80052-167-2.

Zhou, K. C. (1984). An Introduction to English Phonetics. Shanghai Foreign Language Education Press. Shanghai. ISBN7-81009-030-5.

Zhou, L. (1995). Arabic Linguistics. First edition. Foreign Language Teaching and Research Press. Beijing. ISBN7-5600-1018-0/H·553.

Zhu, M. X. (1992). The Speech Sound Technology of Computer. Beihang University Press. Beijing. ISBN7-81012-321-1/TP.067

\section{Copyrights}

Copyright for this article is retained by the author(s), with first publication rights granted to the journal.

This is an open-access article distributed under the terms and conditions of the Creative Commons Attribution license which permits unrestricted use, distribution, and reproduction in any medium, provided the original work is properly cited. 